\title{
Histomorphometric evaluation of tibial subchondral bone after moderate running in male and female Wistar rats
}

\author{
M.N. Nazem®, M. Sahebozamani, R. Mosavian Naeini, S.M. Sajjadian \\ Shahid Bahonar University of Kerman, Iran
}

[Received: 7 May 20219; Accepted: 30 November 2019]

\begin{abstract}
Background: Exercise has been shown to be beneficial to the skeleton, in both humans and animals. This study was done to test the sex-related difference in the risk of osteoarthritis $(O A)$ of the knee joint and also on the subchondral bone after moderate running exercise.

Materials and methods: Forty male and female Wistar rats were randomly assigned to four equal groups ( 2 male and 2 female groups) in the same condition. Ten animals of each sex were selected as control groups, while running exercises were performed in remaining 20 male and female rats, using a motor treadmill to motivate rats to run daily distances of $1 \mathrm{~km}$ at 5 days/week within 6 weeks. On day 43, all animals were sacrificed and the knee articular cartilage and also histomorphometric parameters of subchondral bone and mid shaft of tibia were evaluated. Results: Results showed mild OA in both male and female runner groups. Results in male runner rats were significantly lesser than that in female runners. On the other hand, the difference in female runner group showed significant changes in comparison with other groups in histomorphometric parameters in tibia.

Conclusions: Obtained results showed that the development of knee OA and subchondral bone changes may be related to the sex differences. Although there was no synovitis in male runners, female runner group showed mild hyperaemia dropsy with a moderate synovitis in this region. (Folia Morphol 2021; 80, 1: 127-132)
\end{abstract}

Key words: histomorphometry, running, subchondral bone, tibia, rat

\section{INTRODUCTION}

According to human [7] and animal [12] studies, exercise and mechanical loading are beneficial to the skeleton. Despite the obvious effects of high mechanical loading on the skeleton, the capacity of skeleton to maintain the exercise-induced bone gain remains unknown [27].

Based on multiple studies with experimental models, increased mechanical loading or physical activity triggers bone formation and growth and has positive effects on the skeleton $[9,14,29]$. Studies show that running exercise increases cortical and cancellous tibial bone mass in aged female rats [2].

The articular cartilage health is dependent on the mechanical features of its subchondral bone. It has been shown that bone changes are associated with osteoarthritis (OA) [25]. The architecture of subchondral metaphyseal bone is not uniform. The organisation of trabecular sheets is in a way that they can transfer stress from the overlying layers of articular cartilage, subchondral plate, and calcified cartilage to metaphyseal cortices $[15,18]$.

Address for correspondence: M.N. Nazem, Associate Professor of Anatomy, Histology and Embryology, Department of Basic Sciences, School of Veterinary Medicine, Shahid Bahonar University of Kerman, Pazhuhesh Square, Kerman, Iran. P.O. Box: 76169133, tel: +98 3431322954, fax: +98 3433257447, e-mail: nnazem@uk.ac.ir; nasernazem@yahoo.com 
In this study, we aimed to evaluate the effects of moderate running exercise on the subchondral bone of tibia in the knee joint, histological assessment and grading of the synovial membrane changes and to conduct a histomorphometric assessment of the mid-shaft of tibia.

\section{MATERIALS AND METHODS}

All procedures involving the experimental use of animals were approved by the Animal Ethics Committee, a branch of the Research Council of the Veterinary School in Shahid Bahonar University, Kerman Province, Iran.

Forty adult male and female Wistar rats (9-10 weeks of age) with no significant difference in their body weight ( $251.59 \pm 12.31 \mathrm{~g})$ were randomly divided into four equal groups. All rats were allowed a 5-day adaptation period in a room with controlled conditions (temperature $22-25^{\circ} \mathrm{C}$ and humidity $60-70 \%$ ) before starting the experiment [28]. Ten animals of each sex were selected as male and female control groups while running exercises were performed in remaining 20 male and female rats for 6 weeks on a motor-driven rodent treadmill (Model T510, DRI Co., Taiyuan, Taiwan), speed of $20 \mathrm{~m} / \mathrm{min}$ with inclination of $18^{\circ}$ for 60 minutes each day on 5 days of every week.

During the exercise period, all animals were fed a standard diet (Javaneh Khorasan Co., Iran).

On day 43, all control and runner rats were sacrificed by overdose injection of pentobarbital sodium $(100 \mathrm{mg} / 100 \mathrm{~g}$ body weight, intraperitoneal injection). The body weight was recorded. Then whole of both knee joints were taken and fixed in $10 \%$ buffered formaldehyde for 1 day and then was placed in a new solution of $10 \%$ buffered formaldehyde for 10 days. Decalcification in $10 \%$ formic acid solution was followed by embedding of the complete knee joints in this solution for 50 days. $5 \mu \mathrm{m}$ thickness serial sections of frontal whole left knee joint were prepared in order to obtain the thickest cartilage section from the lateral condyle of the tibia. Obtained thickest sections of each tibia were stained with haematoxylin-eosin method. Sections were evaluated using the Mankin's scheme [19]. We only differed safranin-O staining with haematoxylin-eosin method. The histological evaluation system for OA was classified into four categories: Mankin score of 0 , no OA; Mankin scores of $1-5$, mild OA; scores of 6-10, moderate $O A$; and scores of 11-14, severe OA [24]. In order to histological grading of synovial layer changes, Krenn et al.'s method was used. Based on their results, 0 to 1 corresponds to no synovitis, 2 to 4 to a slight synovitis, 5 to 6 to a moderate synovitis, and 7 to 9 to a strong synovitis [16].

Histomorphometric analyses were taken on sections using method described by Renner et al. [26]. Thickness from subchondral bone to articular surface was measured at the middle of lateral condyle. In each section, chondrocyte cells were counted within a $120,000 \mu \mathrm{m}^{2}$ area including both calcified layer and articular surface [26].

All right knee joints were opened and examined for gross knee articular surface evaluation.

On the other hand, the right tibia was dissected out. The tibia was cut into two equal parts. Thus, the proximal half of tibia was collected. The samples were proceeding similar to the left ones. Serial longitudinal sections were cut with a rotary microtome and mounted on saline-coated slides glass after preparation of histological specimens. Before staining, the sections were deparaffinised in $100 \%$ xylene and rehydrated in graded ethanol [1]. The longitudinal sections of the proximal tibia, between medial and lateral intercondyloid spines, and transverse sections of the mid shaft of tibia were cut at $5-\mu \mathrm{m}$ thickness with a microtome.

Histomorphometric measurements of the cancellous bone of the proximal tibia and the cortical bone of the tibial shaft were examined using an Olympus light microscope and photomicrographs were taken by an attached eyepiece camera (Dino-eye, AM-7023, $5 \mathrm{Mp}$, Taiwan) on $40 \times$ and $100 \times$ magnification. Ten random fields were selected in each slide.

Histomorphometric parameters were described according to the American Society for Bone and Mineral Research nomenclature committee [30].

The histomorphometric parameters evaluated for cancellous bone were bone volume (BV; the percentage of occupied area by calcified bone in relation to the total area), ratio of BV to TV which included trabecular bone volume (BV/TV), trabecular number ( $\mathrm{TbN}$; number of trabeculae that a line through a trabecular compartment would hit per millimetre of its length), trabecular thickness ( $\mathrm{TbTh}$; the thickness of 40 complete trabecular bone packet on straight, rodlike trabecular structures in each section), osteoblast surface $(\mathrm{ObS} / \mathrm{BS}$; per cent of bone surface occupied by osteoblasts and osteoclast surface (OcS/BS; per cent of bone surface occupied by osteoclasts) [17, 30].

The histomorphometric parameters evaluated for tibial shaft were total tissue area (Tt Ar), marrow cavity area (Mc $\mathrm{Ar}$ ) and cortical bone area ( $\mathrm{Cb} \mathrm{Ar})$. These pa- 
Table 1. The changes (mean \pm standard error) of weight $(\mathrm{g})$ at the first day and end day of the exercise period

\begin{tabular}{llcc}
\hline Groups & & $\begin{array}{c}\text { First day } \\
\text { of exercise }\end{array}$ & $\begin{array}{c}\text { End day } \\
\text { of exercise }\end{array}$ \\
\hline Control & Male & $254.14 \pm 18.54$ & $256.2 \pm 16.82$ \\
& Female & $248.32 \pm 12.32$ & $254.14 \pm 14.81$ \\
\multirow{2}{*}{ Runner } & Male & $253.32 \pm 21.08$ & $241.37 \pm 17.03$ \\
& Female & $249.23 \pm 16.01^{\mathrm{a}}$ & $230.08 \pm 17.01^{\mathrm{b}}$ \\
\hline
\end{tabular}

$a, b$ ln the same row with no common superscript show significant difference $(p<0.05)$.
Table 2. Mankin's histopathology and synovial gradings (mean \pm standard error) of distal extremity of lateral condyle of tibia

\begin{tabular}{|c|c|c|c|c|}
\hline & \multicolumn{2}{|c|}{ Male } & \multicolumn{2}{|c|}{ Female } \\
\hline & Control & Runner & Control & Runner \\
\hline Mankin* & $0^{\mathrm{a}}$ & $1.02 \pm 0.38^{a}$ & $0^{\mathrm{a}}$ & $5.12 \pm 0.89^{b}$ \\
\hline Synovial layer** & $0^{a}$ & $0.8 \pm 0.36^{a}$ & $0^{\mathrm{a}}$ & $5.21 \pm 0.32^{b}$ \\
\hline
\end{tabular}

$\mathrm{a}, \mathrm{b}$ the same row with no common superscript show significant difference $(\mathrm{p}<0.05)$, ${ }^{*} 0$ : No osteoarthritis; 1-5: Mild osteoarthritis; $6-10$ : Moderate osteoarthritis; 11-14: Severe osteoarthritis, ${ }^{*} 0$ to 1 corresponds to no synovitis, 2 to 4 to a slight synovitis, 5 to 6 to a moderate synovitis, and 7 to 9 to a strong synovitis.

Table 3. Bone histomorphometry (mean \pm standard error) of cancellous and cortical parts of tibia

\begin{tabular}{|c|c|c|c|c|}
\hline & \multicolumn{2}{|c|}{ Male } & \multicolumn{2}{|c|}{ Female } \\
\hline & Control & Runner & Control & Runner \\
\hline \multicolumn{5}{|c|}{ Cancellous part } \\
\hline $\mathrm{TbN}$ & $6.75 \pm 0.75^{\mathrm{a}}$ & $10.43 \pm 0.75^{b}$ & $7.25 \pm 0.63^{\mathrm{a}}$ & $19.25 \pm 0.75^{c}$ \\
\hline $\mathrm{TbTh}[\mu \mathrm{m}]$ & $43.32 \pm 5.47^{a, b}$ & $46.54 \pm 4.7^{\mathrm{a}, \mathrm{b}}$ & $39.41 \pm 4.01^{\mathrm{a}}$ & $54.93 \pm 4.86^{b}$ \\
\hline BV/TV [\%] & $58.32 \pm 5.71^{\mathrm{a}}$ & $60.3 \pm 5.32^{\mathrm{a}}$ & $55.43 \pm 4.91^{\mathrm{a}}$ & $72.83 \pm 6.32^{b}$ \\
\hline 0cS/BS [\%] & $8.42 \pm 0.6^{a}$ & $8.12 \pm 0.52^{\mathrm{a}, \mathrm{b}}$ & $8.51 \pm 0.61^{\mathrm{a}}$ & $7.82 \pm 0.42^{b}$ \\
\hline ObS/BS [\%] & $53.41 \pm 5.12^{\mathrm{a}}$ & $56.02 \pm 4.01^{\mathrm{a}, \mathrm{b}}$ & $51.07 \pm 4.92^{\mathrm{a}}$ & $62.41 \pm 3.12^{b}$ \\
\hline \multicolumn{5}{|l|}{ Cortical part } \\
\hline $\operatorname{Tt} \operatorname{Ar}\left[\mathrm{mm}^{2}\right]$ & $541.48 \pm 27.32^{a, b}$ & $558.92 \pm 31.27^{\mathrm{a}, \mathrm{b}}$ & $524.61 \pm 19.27^{\mathrm{a}}$ & $561.58 \pm 23.97^{b}$ \\
\hline $\operatorname{Mc} \operatorname{Ar}\left[\mathrm{mm}^{2}\right]$ & $172.11 \pm 10.27^{\mathrm{a}, \mathrm{c}}$ & $161.92 \pm 9.81^{a}$ & $180.01 \pm 14.31^{b c}$ & $161.14 \pm 9.97^{a}$ \\
\hline $\mathrm{Cb} \operatorname{Ar}\left[\mathrm{mm}^{2}\right]$ & $328.31 \pm 24.03^{\mathrm{a}, \mathrm{b}}$ & $333.12 \pm 19.81^{\mathrm{a}, \mathrm{b}}$ & $312.42 \pm 16.81^{\mathrm{a}}$ & $348.91 \pm 23.14^{b}$ \\
\hline
\end{tabular}

The abbreviations are mentioned in material and methods. ${ }^{a, b, c}$ In the same row with no common superscript show significant difference $(p<0.05)$.

rameters were chosen based on a previous study [1]. The results were obtained by using an Olympus light microscope and an attached eyepiece digital lens (Dino-eye, AM-7023, 5Mp, Taiwan).

\section{Statistical analysis}

Results were expressed as means \pm standard error using the software SPSS 16 (version 16, Chicago, USA). Statistical analysis was carried out using an independent T-test and one-way ANOVA. Also the Tukey's test was used for post hoc analysis with significance set at $p<0.05$.

\section{RESULTS}

\section{Weight}

In the female exercise group, body weight decreased markedly, compared to the control group at the end of the intervention (Table 1).

\section{Bone histomorphometry and histopathology}

Knee joint: Gross findings. The surface of the femoral cartilage was found to be smooth in both female and male control groups, whereas in four male runner rats, the articular surface was irregular after the sixth week. In the female exercise group, superficial cartilage irregularities were detected in the trochlea and femoral condyles.

\section{Histological finding}

Our findings were indicative of mild $O A$ in both female and male exercise groups. The results in male runner rats were less significant than those of female runners (Table 2).

Based on the findings, the synovial layer was normal in both female and male control groups. Most male runner rats in the control group had a normal condition.

There was no synovitis in male runners. However, the synovial joint seemed almost coarse and thick in the female runner group, with mild hyperaemia dropsy. There was also a moderate synovitis in this group (Table 2).

In the female exercise group, OcS/BS\% was found to be significantly lower than that of the female control group (Table 3). 
In the female runner group, ObS/BS\% showed a significant increase compared to the female controls (Table 3).

The trabecular number (TbN) increased significantly in both female and male exercise groups, compared to the controls. On the other hand, the trabecular thickness (Tt Ar) difference between female runner and control rats was significant (Table 3).

The BV/TV ratio was higher in male and female exercise groups, compared to the corresponding control groups. The difference between female runner rats was significant (Table 3).

A greater total tissue area ( $\mathrm{Tt} \mathrm{Ar}$ ) and a smaller marrow cavity area (Mc Ar) were found in both exercise groups, compared to the controls. The difference between the control and runner female groups was significant (Table 3). On the other hand, the cortical bone area ( $\mathrm{Cb} \mathrm{Ar}$ ) was significantly greater in the female exercise group in comparison with the corresponding control group (Table 3 ).

\section{DISCUSSION}

In the present study, moderate-intensity running was found to damage the articular cartilage; therefore, it is considered a strenuous exercise, especially for females. Based on our findings, in moderate-intensity exercise, women experience more cartilage changes, compared to men with a similar body weight. According to previous research, the cartilage volume of normal men is $33 \%$ to $42 \%$ larger than that of normal women, as indicated in radiographic analyses [3].

Moreover, Jones et al. [13] showed that the knee cartilage of men is significantly more than that of females. It was concluded that sex differences in cartilage development could explain the variations in $\mathrm{OA}$ of the knee in later stages of life. In addition, Faber et al. [6] reported that after adjustments for height and body weight, gender differences did not influence cartilage volume. Nevertheless, it was reported that body and bone size had significant effects on this difference, suggesting a direct relationship between cartilage volume and bone size [5, 13].

Evidence suggests the thicker cartilage of men, compared to women [22]. In contrast, no gender-related effects have been reported after adjustments for body weight and tibial head diameter [4]. Some significant changes were detected in rats with matching age and body weight; therefore, other parameters might explain gender differences
Previous studies show that running exercise decreases fat mass $[7,20]$. Our obtained weight results were similar to this study.

The main bone function is to bear gravity-induced mechanical forces and muscle contraction and to facilitate efficient body locomotion [23]. In a study by Iwamoto et al. [11], to preserve the positive effects of exercise (for 8 weeks), continuous exercise was found to be necessary. To explain the mechanism through which exercise leads to increased bone mass on histomorphometry, Yeh et al. [31] showed that suppressed bone resorption and improved bone formation might be responsible for the positive effects of exercise on the cancellous bones of tibia in aged female rats. Moreover, Chen et al. [2] showed that exercise leads to cortical bone mass accumulation in the tibia of aged female rats. Our data demonstrated that moderate running exercise resulted in significant increases in both cancellous and cortical bone mass (BV/TV and $\mathrm{Ct}$ $A r$, respectively) in a weight-bearing long bone, the tibia, in the female rats. The results of OcS/BS and $\mathrm{ObS} / \mathrm{BS}$ percentage in female rats indicated that the response of cancellous bone to exercise differs according to the magnitude of mechanical loading to the bone. There is a threshold in mechanical loading to the bones when cancellous bone mass increases, with bone formation exceeding bone resorption (Table 3 ). These findings are consistent with the researchers' hypothesis $[10,11]$.

Joint tissues can sustain minimal damage without progressive change. It is known that cumulative micro-damage can lead to bone remodelling, stiffening, and cartilage lesion progression. Peak dynamic forces applied at a high rate cause subchondral bone remodelling and stiffening [8]. In this study, cortical and cancellous bone areas increased significantly in the tibia due to exercise. It should be noted that exercise duration and intensity were probably ideal for increasing cancellous bone mass in our study. Our results also demonstrated that the effect of exercise in females was more significant than males.

In a previous study, it was reported that moderate running exercise significantly increased cortical and cancellous bone mass in tibia [11]; our findings are in consistence with this study. This finding might be attributed to the position of tibia and mechanical loading on the tibia during treadmill running $[11,21]$. Moreover, Chang et al. [1] showed that the cancellous bone response to exercise changes relative to the bone mechanical loading. This finding is consistent 
with a previous study, which indicated the positive site-specific effect of treadmill exercise on bone mineral density, with predominant weight-bearing sites [11].

\section{CONCLUSIONS}

Our results showed the moderate exercises are more harmful in females than males. Also subchondral bone changes in females may be more visible than in males.

\section{Acknowledgements}

This research was financially supported by the research council of Shahid Bahonar University of Kerman (No: 1.7.1395). The authors would like to thank Mr. Mazhab Jaafari for providing histological laboratory methods and histology slides. The authors declared no conflict of interests.

\section{REFERENCES}

1. Chang $\mathrm{TK}$, Huang $\mathrm{CH}$, Huang $\mathrm{CH}$, et al. The influence of long-term treadmill exercise on bone mass and articular cartilage in ovariectomized rats. BMC Musculoskelet Disord. 2010; 11: 185, doi: 10.1186/1471-2474-11-185, indexed in Pubmed: 20727126.

2. Chen MM, Yeh JK, Aloia JF, et al. Effect of treadmill exercise on tibial cortical bone in aged female rats: a histomorphometry and dual energy $x$-ray absorptiometry study. Bone. 1994; 15(3): 313-319, doi: 10.1016/8756-3282(94)90294-1, indexed in Pubmed: 8068453.

3. Ding $C$, Cicuttini F, Scott F, et al. Sex differences in knee cartilage volume in adults: role of body and bone size, age and physical activity. Rheumatology (Oxford). 2003; 42(11): 1317-1323, doi: 10.1093/rheumatology/keg374, indexed in Pubmed: 12810930.

4. Eckstein F, Cicuttini F, Raynauld JP, et al. Magnetic resonance imaging (MRI) of articular cartilage in knee osteoarthritis (OA): morphological assessment. Osteoarthritis Cartilage. 2006; 14 Suppl A: A46-A75, doi: 10.1016/j. joca.2006.02.026, indexed in Pubmed: 16713720.

5. Eckstein F, Winzheimer M, Hohe J, et al. Interindividual variability and correlation among morphological parameters of knee joint cartilage plates: analysis with three-dimensional MR imaging. Osteoarthr Cartil. 2001; 9(2): 101-111, doi: 10.1053/joca.2000.0365, indexed in Pubmed: 11237657.

6. Faber SC, Eckstein F, Lukasz $\mathrm{S}$, et al. Gender differences in knee joint cartilage thickness, volume and articular surface areas: assessment with quantitative three-dimensional MR imaging. Skeletal Radiol. 2001; 30(3): 144-150, doi: 10.1007/s002560000320, indexed in Pubmed: 11357452.

7. Howe T, Shea B, Dawson L, et al. Exercise for preventing and treating steoporosis in postmenopausal women. Cochrane Data Sys Rev. 2015; 6(7): CD000333, doi: 10.1002/14651858.CD000333.pub2, indexed in Pubmed: 21735380 .
8. Hudelmaier M, Glaser C, Hausschild A, et al. Effects of joint unloading and reloading on human cartilage morphology and function, muscle cross-sectional areas, and bone density: a quantitative case report. J Musculoskelet Neuronal Interact. 2006; 6(3): 284-290, indexed in Pubmed: 17142951.

9. Iwamoto J, Sato Y, Takeda T, et al. Effectiveness of exercise for osteoarthritis of the knee: a review of the literature. World J Orthop. 2011; 2(5): 37-42, doi: 10.5312/wjo. v2.i5.37, indexed in Pubmed: 22474634.

10. Iwamoto J, Takeda T, Ichimura S. Effect of exercise on tibial and lumbar vertebral bone mass in mature osteopenic rats: bone histomorphometry study. J Orthop Sci. 1998; 3(5): 257-263, doi: 10.1007/s007760050051, indexed in Pubmed: 9732560.

11. Iwamoto J, Yeh JK, Aloia JF, et al. Effect of deconditioning on cortical and cancellous bone growth in the exercise trained young rats. J Bone Miner Res. 2000; 15(9): 1842-1849, doi: 10.1359/jbmr.2000.15.9.1842, indexed in Pubmed: 10977004.

12. Järvinen $T L$, Kannus $P$, Sievänen $H$, et al. Effects of remobilization on rat femur are dose-dependent. Scand J Med Sci Sports. 2001; 11(5): 292-298, doi: 10.1034/j.16000838.2001.110507.x, indexed in Pubmed: 11696214.

13. Jones $G$, Glisson $M$, Hynes $K$, et al. Sex and site differences in cartilage development: a possible explanation for variations in knee osteoarthritis in later life. Arthritis Rheum. 2000; 43(11): 2543-2549, doi: 10.1002/1529-0131(200011)43:11<2543::AID-ANR23>3.0.CO;2-K, indexed in Pubmed: 11083279.

14. Joo YI, Sone $T$, Fukunaga $M$, et al. Effects of endurance exercise on three-dimensional trabecular bone microarchitecture in young growing rats. Bone. 2003; 33(4): 485-493, doi: 10.1016/s8756-3282(03)00212-6, indexed in Pubmed: 14555251.

15. Kenneth D, Brandt M. Osteoarthritis. In: Kasper, Braunwald, Fauci. Hauser, Longo, Jameson, Editors: Harrison's priniciples of internal medicine. 15th ed. USA. Bmc Graw - Hill Incl, USA 2005: 2036-2045.

16. Krenn V, Morawietz L, Häupl T, et al. Grading of chronic synovitis - a histopathological grading system for molecular and diagnostic pathology. Path Res Prac. 2002; 198(5): 317-325, doi: 10.1078/0344-0338-5710261.

17. Kulak CA, Dempster DW. Bone histomorphometry: a concise review for endocrinologists and clinicians. Arq Bras Endocrinol Metabol. 2010; 54(2): 87-98, doi: 10.1590/ s0004-27302010000200002, indexed in Pubmed: 20485895.

18. Lee YJ, Park JiAh, Yang SH, et al. Evaluation of osteoarthritis induced by treadmill-running exercise using the modified Mankin and the new OARSI assessment system. Rheumatol Int. 2011; 31(12): 1571-1576, doi: 10.1007/s00296-0101520-4, indexed in Pubmed: 20490805.

19. Mankin H, Dorfman H, Lippiello L, et al. Biochemical and metabolic abnormalities in articular cartilage from osteo-arthritic human hips. J Bone Joint Surg Am. 1971; 53(3): 523-537, doi: 10.2106/00004623-19715303000009.

20. Morseth B, Emaus N, Jørgensen L. Physical activity and bone: The importance of the various mechanical stimuli 
for bone mineral density. A review. Norsk Epidemiologi. 2011; 20(2), doi: 10.5324/nje.v20i2.1338.

21. Mosavian NR, Sahebozamani M, Nazem MN. Effect of moderate treadmill exercise on hip osteoarthritis in male and female Wistar rats. Novel Biomed. 2017; 4: 177-184.

22. Ni GX, Zhan LQ, Gao MQ, et al. Matrix metalloproteinase-3 inhibitor retards treadmill running-induced cartilage degradation in rats. Arthritis Res Ther. 2011; 13(6): R192, doi: 10.1186/ar3521, indexed in Pubmed: 22114772.

23. Pajamäki I, Kannus P, Vuohelainen T, et al. The bone gain induced by exercise in puberty is not preserved through a virtually life-long deconditioning: a randomized controlled experimental study in male rats. J Bone Miner Res. 2003; 18(3): 544-552, doi: 10.1359/jbmr.2003.18.3.544, indexed in Pubmed: 12619940.

24. Pap G, Eberhardt R, Stürmer I, et al. Development of osteoarthritis in the knee joints of Wistar rats after strenuous running exercise in a running wheel by intracranial self-stimulation. Path Res Prac. 1998; 194(1): 41-47, doi: 10.1016/s0344-0338(98)80010-1.

25. Radin EL, Rose RM. Role of subchondral bone in the initiation and progression of cartilage damage. Clin Orthop Relat Res. 1986(213): 34-40, indexed in Pubmed: 3780104
26. Renner AF, Carvalho E, Soares E, et al. The effect of a passive muscle stretching protocol on the articular cartilage. Osteoarthr Cartil. 2006; 14(2): 196-202, doi: 10.1016/j.joca.2005.08.011, indexed in Pubmed: 16243547.

27. Seeman E. An exercise in geometry. J Bone Miner Res. 2002; 17(3): 373-380, doi: 10.1359/jbmr.2002.17.3.373, indexed in Pubmed: 11874229.

28. Seyyedin S, Nazem MN. Histomorphometric study of the effect of methionine on small intestine parameters in rat: an applied histologic study. Folia Morphol. 2017; 76(4): 620-629, doi: 10.5603/fm.a2017.0044, indexed in Pubmed: 28553855.

29. Tromp AM, Bravenboer N, Tanck E, et al. Additional weight bearing during exercise and estrogen in the rat: the effect on bone mass, turnover, and structure. Calcif Tissue Int. 2006; 79(6): 404-415, doi: 10.1007/s00223-006-0045-z, indexed in Pubmed: 17160577.

30. Vidal B, Pinto A, Galvão MJ, et al. Bone histomorphometry revisited. Acta Reumatol Port. 2012; 37(4): 294-300, indexed in Pubmed: 24126421.

31. Yeh J, Aloia J, Chen M, et al. Influence of exercise on cancellous bone of the aged female rat. J Bone Miner Res. 1993; 8(9): 1117-1125, doi: 10.1002/jbmr.5650080913. 